Dear Author,

Please correct your galley proofs carefully and return them no more than three days after the page proofs have been received.

If you have not used the PXE system before, please view the Tutorial before checking your proofs:

http://wileypxe.aptaracorp.com/pxewileyvch/ UserDocument/UserGuide/WileyPXE5 Author Instructions.pdf

Please note any queries that require your attention. These are indicated with red Qs in the pdf or highlighted as yellow queries in the "Edit" window.

Please pay particular close attention to the following, as no further corrections can be made once the article is published online:

- Names of all authors present and spelled correctly

- Titles of authors are correct (Prof. or Dr. only: please note, Prof. Dr. is not used in the journals)

- Addresses of all authors and e-mail address of the corresponding author are correct and up-to-date

- Funding bodies have been included and grant numbers are accurate
- The Title of the article is OK

- All figures are correctly included

- Equations are typeset correctly

Note that figure resolution in the PXE system is deliberately lower to reduce loading times. This will be optimized before the article is published online.

Please send any additional information, such as figures or other display items, to advmatinterfaces@wiley-vch.de, and please also indicate this clearly in the PXE "Edit" window by inserting a comment using the query tool.

Reprints may be ordered by filling out the accompanying form.

Return the reprint order form by e-mail with the corrected proofs, to Wiley- VCH: advmatinterfaces@wiley-vch.de

Please limit corrections to errors already in the text. Costs incurred for any further changes will be charged to the author, unless such changes have been agreed upon by the editor.

The editors reserve the right to publish your article without your corrections if the proofs do not arrive in time. Note that the author is liable for damages arising from incorrect statements, including misprints. 
Reprint Order Form 2017

\section{Short DOI: admi.}

Please send me and bill me for

no. of Reprints via $\square$ airmail (+ 25 Euro)

Please send me and bill me for a

high-resolution PDF file (330 Euro).

My Email address:

Please note: It is not permitted to present the PDF file on the internet or on company homepages.

\section{Information regarding VAT}

Please note that from German sales tax point of view, the charge for Reprints, Issues or Posters is considered as "supply of goods" and therefore, in general, such delivery is a subject to German sales tax. However, this regulation has no impact on customers located outside of the European Union. Deliveries to customers outside the Community are automatically tax-exempt. Deliveries within the Community to institutional customers outside of Germany are exempted from the German tax (VAT) only if the customer provides the supplier with his/her VAT number. The VAT number (value added tax identification number) is a tax registration number used in the countries of the European Union to identify corporate entities doing business there. It starts with a country code (e.g. FR for France, GB for Great Britain) and follows by numbers.

VAT no.:

(Institutes / companies in EU countries only)

Purchase Order No.:
Editorial office:

Wiley-VCH Verlag

Boschstraße 12, 69469 Weinheim

Germany

Tel.: (+49) 3047031331

Fax: (+49) 6201606500

Email: advmatinterfaces@wiley-vch.de

Delivery address / Invoice address:

Name of recipient, University, Institute, Street name and Street number, Postal Code, Country

\section{Date and Signature:}

Credit Card Payment (optional) -You will receive an invoice.

\section{VISA, MasterCard, AMERICAN EXPRESS}

Please use the Credit Card Token Generator located at the website below to create a token for secure payment. The token will be used instead of your credit card number.

\section{Credit Card Token Generator:}

https://www.wiley-vch.de/editorial production/index.php

Please transfer your token number to the space below.

Credit Card Token Number

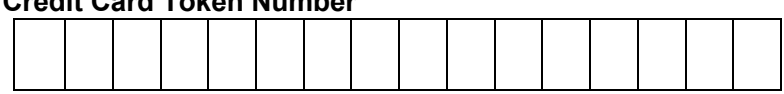

Price list for reprints (The prices include mailing and handling charges. All Wiley-VCH prices are exclusive of VAT)

\begin{tabular}{|c|c|c|c|c|c|c|}
\hline No. of pages & 50 copies & 100 copies & $\begin{array}{l}\text { Price (in E } \\
150 \text { copies }\end{array}$ & $\begin{array}{l}\text { for orders of } \\
200 \text { copies }\end{array}$ & 300 copies & 500 copies \\
\hline $\begin{array}{l}1-4 \\
5-8\end{array}$ & $\begin{array}{l}345 \\
490\end{array}$ & $\begin{array}{l}395 \\
573\end{array}$ & $\begin{array}{l}425 \\
608\end{array}$ & $\begin{array}{l}445 \\
636\end{array}$ & $\begin{array}{l}548 \\
784\end{array}$ & $\begin{array}{c}752 \\
1077\end{array}$ \\
\hline $9-12$ & 640 & 739 & 786 & 824 & 1016 & 1396 \\
\hline $13-16$ & 780 & 900 & 958 & 1004 & 1237 & 1701 \\
\hline $17-20$ & 930 & 1070 & 1138 & 1196 & 1489 & 2022 \\
\hline $\begin{array}{c}\text { for every additional } 4 \\
\text { pages }\end{array}$ & 147 & 169 & 175 & 188 & 231 & 315 \\
\hline
\end{tabular}

Wiley-VCH Verlag GmbH \& Co. KGaA; Location of the Company: Weinheim, Germany;

Trade Register: Mannheim, HRB 432833, Chairman of the Board: Mark Allin

General Partner: John Wiley \& Sons GmbH, Location: Weinheim, Germany

Trade Register Mannheim, HRB 432296, Managing Director: Sabine Steinbach 


\section{FULL PAPER}

$\mathbf{x X X X}$

P. Gisbert-Quilis, M. Masetti, J. Morla-Folch, J. Fitzgerald, N.

Pazos-Perez, E. Garcia-Rico, V. Giannini, R. A. Alvarez-Puebla,* L. Guerrini* $. x-x x$

The Structure of Short and Genomic DNA at the Interparticle Junctions of Cationic Nanoparticles

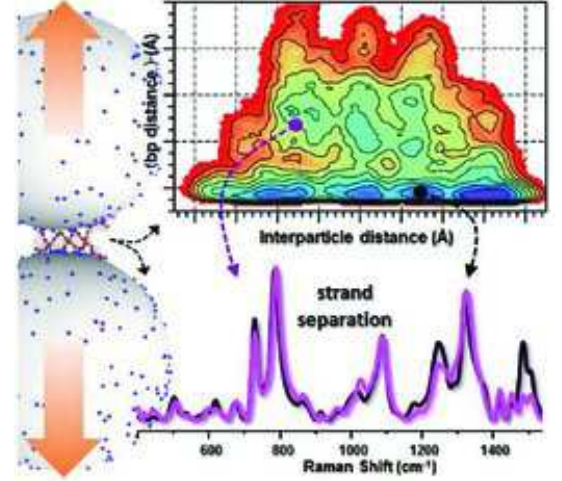

Unraveling the mechanism of DNA unzipping mediated by interaction with cationic nanoparticles. Key questions regarding the cooperative role of nanoparticle clustering, nanoparticle surface charge, and nucleic acids properties are directly addressed by combining plasmon-based spectroscopies and theoretical simulations with no need of external intercalating dyes or prelabeling steps. 


\title{
The Structure of Short and Genomic DNA at the Interparticle Junctions of Cationic Nanoparticles
}

\author{
Patricia Gisbert-Quilis, Matteo Masetti, Judit Morla-Folch, Jamie Fitzgerald, \\ Nicolas Pazos-Perez, Eduardo Garcia-Rico, Vincenzo Giannini, \\ Ramon A. Alvarez-Puebla, * and Luca Guerrini*
}

Current understanding of the mechanisms underlying noncovalent interactions between native DNA and nanoparticles, as well as their impact on the double-helix structure, is far from providing a comprehensive view. It is known that these interactions are largely defined by the physicochemical properties of the metal/liquid interface, in particular by the nanoparticle surface charge. Remarkably, while DNA unzipping upon binding with cationic nanoparticles is reported, the exact determinants of this structural perturbation remain unclear. Herein, plasmon-based spectroscopies (surface-enhanced Raman scattering (SERS) and surface-plasmon resonance) and theoretical simulations are combined to directly investigate the role of the cooperative binding of cationic nanoparticles with different surface charges on the structural integrity of a large variety of DNAs. The intrinsic nature of the SERS effect unlocks the possibility of selectively examining the impact of nanoparticle clustering on the duplex structure over a wide degree of colloidal aggregation and without the need of external intercalating dyes or strand labeling. This extensive work provides new fundamental insights into the interaction between nucleic acids and nanoparticles, addressing key questions regarding the role played by multiple variables such as the nanoparticle surface charge, the DNA-mediated cluster size and geometry, and nucleic acids' length, composition, and conformational properties.

P. Gisbert-Quilis, J. Morla-Folch, Dr. N. Pazos-Perez, Prof. R. A. Alvarez-Puebla, Dr. L. Guerrini

Department of Physical Chemistry

Universitat Rovira I Virgili

Carrer de Marcel.lí Domingo s/n, 43007, Tarragona, Spain

Dr. M. Masetti

Department of Pharmacy and Biotechnology

Alma Mater Studiorum-Università di Bologna

Via Belmeloro 6, 40126, Bologna, Italy

J. Fitzgerald, Dr. V. Giannini

Department of Physics

Imperial College

London, SW7 2AZ, UK

Dr. E. Garcia-Rico

Fundacion de Investigacion HM Hospitales

Madrid San Bernardo 101, 28015, Spain

Dr. E. Garcia-Rico

Centro Integral Oncologico Clara Campal (CIOCC)

Madrid Oña 10, 28050, Spain

Dr. E. Garcia-Rico

Department of Medical Oncology

Hospital Universitario HM Torrelodones

Castillo de Olivares s/n, 28250, Torrelodones, Spain

\section{Introduction}

Integration of nucleic acids (NAs) and nanoparticles (NPs) has generated a wealth of research in the area of nanotechnology, with particular emphasis on nanostructures with unique physicochemical properties such as noble metal nanoparticles. As a result, an increasing number of NA-based nanotechnologies are continuously emerging, finding a diverse set of applications in biomedicine, sensing, bioelectronics, biocomputing, etc. ${ }^{[1]}$ While countless efforts have been devoted to the study of chemically modified oligonucleotides (mostly, thiolated DNA) cova-

Prof. R. A. Alvarez-Puebla

ICREA

Passeig Lluís Companys 23

, 08010, Barcelona, Spain

Correspondence to: Prof. R. A. Alvarez-Puebla (E-mail: ramon.alvarez@urv.cat), Dr. L. Guerrini (E-mail: luca.guerrini@ctqc.org)

The ORCID identification number(s) for the author(s) of this article can be found under http://dx.doi.org/10.1002/admi.201700724

DOI: 10.1002/admi.201700724 
lently attached to plasmonic nanoparticles, ${ }^{[2]}$ the understanding of the mechanisms involved in the noncovalent interaction between native DNA duplexes (dsDNAs), as well as the impact that such an interaction has on the double-helix structure, is far less explored, and reports in the literature often diverge significantly in their findings. ${ }^{[1,3]}$

The interaction between unmodified dsDNA and nanoparticles is largely determined by the physicochemical properties of the interface, with the nanoparticle surface charge playing a major role in this process. ${ }^{[4]}$ In particular, cationic nanoparticles with sufficiently high surface charge are capable of irreversible interactions with the negatively charged phosphate groups of nucleic acids, leading to DNA damage. ${ }^{[4-f, 5]}$ Partial denaturation of DNA duplexes upon complexation with positively charged nanoparticles was previously revealed mainly by direct monitoring of the hyperchromic shift of the DNA absorption band at $260 \mathrm{~nm}^{[6]}$ and by indirect examination of the optical (absorbance or fluorescence) response of intercalating dyes sensitive to the structures of the bound DNA (i.e., duplex vs single strand). ${ }^{[6 b, c]}$ Interestingly, fluorescence studies as well as molecular dynamics (MD) simulations ${ }^{[6 b]}$ suggested that only high concentrations of nanoparticles can bend and separate DNA strands. This led to the hypothesis that the partial strand separation may only occur via a cooperative action of an ensemble of interacting nanoparticles. However, insertion of intercalating molecules causes itself local structural changes to the duplex, including unzipping of the double helix and lengthening of the strand. ${ }^{[7]}$ Additionally, the near-field interaction of the excited-state fluorophore with the surface plasmon resonances (SPR) of metallic nanoparticles perturbs the optical properties of the dye and, thus, its fluorescence emission in a way that is largely dependent on the properties of the plasmonic nanoparticles and the fluorophore, as well as the distance between the two. ${ }^{[8]}$ On the other hand, UV-vis spectroscopy analysis of DNA-NP complexes can only be performed on soluble complexes, thus restricting the case study to very small nanoparticles and/or large DNA/NP molar ratios when phase separation is avoided. ${ }^{[6 a]}$ To a certain extent, this trend is mirrored in theoretical studies, where the nanoscale size of DNA-NP systems in most cases prevents their direct investigation through atomistic simulations. ${ }^{[9]}$ Thus, to the best of our knowledge, MD simulations have been carried out only for very small nanoparticles, with diameters not exceeding $1.5 \mathrm{~nm}$, so as to keep the problem computationally manageable. ${ }^{[6,10]}$

Within this fragmented picture, a plasmon-assisted spectroscopy such as surface-enhanced Raman scattering (SERS) has the potential to be used as a highly informative, ultrasensitive analytical method for the direct interrogation of nucleic acid/nanoparticle interactions and their impact on the structure of the genetic material. ${ }^{[11]}$ Notably, the largest SERS enhancements usually show a high degree of spatial localization, such as those that take place at the interparticle junctions (i.e., hot spots) of highly interacting nanoparticles in colloidal aggregates. ${ }^{[12]} \mathrm{Un}$ der this scenario, SERS can provide a unique tool to isolate the vibrational signature of those molecules that are trapped at the nanoparticle junctions.

Herein, we combined optical methods (SERS and SPR spectroscopies) and theoretical simulations (MD and finite difference time domain (FDTD)) to directly investigate the structural perturbation on nucleic acids imposed by the binding with cationic silver nanoparticles ( $\approx 22 \mathrm{~nm}$ diameter) with tuned $\zeta$ potential. Remarkably, the intrinsic nature of the SERS effect unlocked the possibility of selectively examining the impact of nanoparticle clustering on the double-helix integrity over a wide degree of aggregation (i.e., DNA/NP ratios) and without the need of exogenous fluorescence reporters. On the other hand, SPR analysis and MS integrated critical complementary information to the indepth characterization of the DNA/NP interactions and the underlying mechanisms. A large set of different duplex structures Q4 with different lengths (from short segments to long genomic chain), base composition, sequence and form were investigated. The results show that, when the nanoparticle $\zeta$ potential is lowered below a certain threshold, the double helixes trapped at the interparticle gaps undergo major unzipping, which is largely independent of the investigated nucleic acids' properties (i.e., the final outcome of the interaction is mostly determined by the electrostatic binding between phosphate groups and surface positive charges). On the other hand, for colloids with fixed $\zeta$ potentials, strand separation preferably occurs in the case of small DNA-mediated clusters (i.e., for very low DNA/NP ratios) and, to a lesser extent, in the presence of a large excess of duplexes (i.e., for very high DNA/NP ratios). In this case, however, the length of the duplex does play a relevant role in determining the pattern of the duplex structural reshaping, as the biomolecular size directly affects the features of the colloidal aggregation. These results are complemented and confirmed by MD-based simulations performed on model systems representing cluster aggregates of realistic size.

\section{Results and Discussion}

This section is organized as follows: first, the chemico-physical properties of the cationic nanoparticles are discussed. Then, we describe the experimental scenario under which the direct SERS analysis of DNA takes place and, by means of computational tools, qualitatively predicts the spatial origin of the SERS signal. This is propaedeutic to the interpretation of the SERS spectra revealing strand separation of both short and genomic DNA duplexes upon interaction with silver cationic nanoparticles. Two main variables of the nanoparticle-induced unzipping process were identified (degree of nanoparticle aggregation and nanoparticle surface charge) and investigated separately by SERS and SPR spectroscopies, as well as via molecular dynamic simulations.

\subsection{Cationic Silver Nanoparticles}

The wet-chemical synthesis of positively charged silver nanoparticles (AgSp) was performed by reducing silver ions with sodium borohydride in the presence of spermine tetrahydrochloride. ${ }^{[11 b]}$ The colloidal synthesis yields nanoparticles of $\approx 22 \mathrm{~nm}$ diameter (Figure S1A,B, Supporting Information), with a localized surface plasmon resonance (LSPR) band centered at $\approx 392 \mathrm{~nm}$ and a $\zeta$ potential of $\approx+40 \mathrm{mV}$ (Figure $1 \mathrm{~A}, \mathrm{~B}$ ). $\zeta$ measurements provide the potential at the start of the diffuse layer which is indirectly related to the surface charge at the nanoparticle surface. ${ }^{[13]}$ Nanoparticle concentration $\left(\approx 1.00 \times 10^{-9} \mathrm{M}\right)$ was determined 

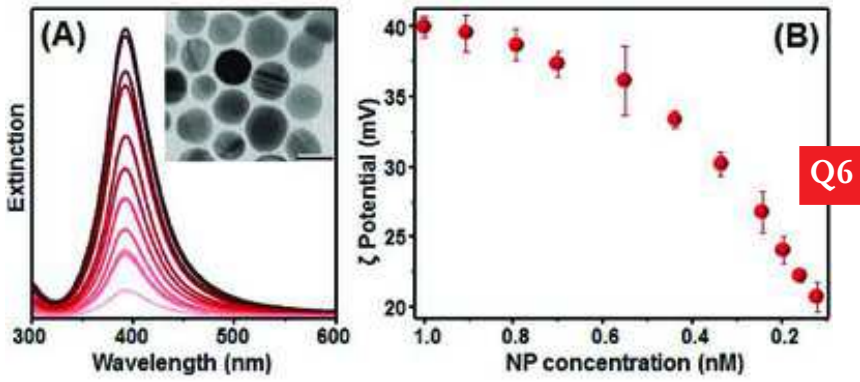

Figure 1. A) Extinction spectra of AgSp colloids at different concentration upon dilution with Milli-Q water. From the top curve to the bottom curve, the estimated nanoparticle concentrations are $1.00 \times 10^{-9}, 0.91 \times 10^{-9}$, $0.79 \times 10^{-9}, 0.70 \times 10^{-9}, 0.55 \times 10^{-9}, 0.44 \times 10^{-9}, 0.34 \times 10^{-9}, 0.24$ $\times 10^{-9}, 0.20 \times 10^{-9}, 0.16 \times 10^{-9}$, and $0.12 \times 10^{-9} \mathrm{M}$. A representative TEM image of the dried nanoparticles is also included (the scale bar represents a length of $20 \mathrm{~nm}$ ). B) $\zeta$ potential values for $\mathrm{AgSp}$ colloids at different concentrations upon dilution with Milli-Q water.

by UV-vis spectroscopy using an extinction coefficient of 54.8 $\times 10^{8} \mathrm{M}^{-1} \mathrm{~cm}^{-1}$ at $392 \mathrm{~nm}$, as obtained from the literature. ${ }^{[14]}$ Spermine molecules are linear quadrivalent cationic polyamines that remain bound to the nanoparticle surfaces via electrostatic interaction mediated by the chloride anions. ${ }^{[15]}$ These ligands provide an overall positive charge to the nanoparticles, and, due to their aliphatic nature, SERS background of the AgSp colloids shows only minimal contribution from the tetramine adlayer (Figure S1C,D, Supporting Information). Dilution of the AgSp colloids with Milli-Q water promotes a progressive reduction in plasmon intensity (Figure 1A) with null or minimal perturbation of the colloidal stability (Figure S2, Supporting Information). At the same time, we register a decrease of $\zeta$ potential (Figure 1B) consistent with the partial desorption of spermine molecules from the metallic surface upon dilution with Milli-Q water. This is further confirmed by the fact that $\zeta$ potential value remains unchanged when nanoparticles are diluted with the colloidal supernatant (Figure S3, Supporting Information).

\subsection{Direct SERS Analysis of DNA: What Do We See?}

We have previously shown that short DNA duplexes of 21 base pairs ( $\mathrm{dsDNA}_{21}$ ) mediate AgSp nanoparticle aggregation acting as electrostatic molecular linkers. ${ }^{[11 b]}$ Under physiological conditions, double-stranded DNA persistence length is of $\approx 150 \mathrm{bp}$, corresponding to $\approx 50 \mathrm{~nm} \cdot{ }^{[16]}$ As a result, dsDNA 21 behaves as a rigid rod of $\approx 7 \mathrm{~nm}$ length and $2 \mathrm{~nm}$ diameter, which is collected at the surface of individual quasispherical nanoparticles of around $22 \mathrm{~nm}$ diameter with minimal bending of the double helix. ${ }^{[10]}$ Molecular dynamics simulations of dsDNA 21 adsorption on AgSp nanoparticles previously indicated that the duplex rapidly adheres onto the metal surface adopting an elongated conformation (for low surface coverage). ${ }^{[11 c]}$ Consequently, interparticle gaps of $\approx 2.5 \mathrm{~nm}$ are generated in $\mathrm{dsDNA}_{21}$-mediated clusters (i.e., the diameter of the duplex rod-like structure sandwiched between spermine molecules bound to the metallic surfaces). It is also worth noting that, in addition to their physical size, DNA molecules coordinated at the metallic surfaces screen further adsorption of unbound duplexes by electrostatic repulsion. ${ }^{[17]}$ The extent of this electrostatic repulsion is larger at low salt concentrations ${ }^{[17]}$ such as for the investigated AgSp colloids $\left(\approx 1-2 \times 10^{-3} \mathrm{M} \mathrm{NaCl}\right.$ resulting from chemicals employed in the nanoparticle synthesis and the PBS buffer of DNA solutions). Thus, the formation of a hypothetical compact monolayer of dsDNA $\mathrm{d}_{21}$, corresponding to $\approx 100$ molecules per particle (based on the average NP diameter and $\mathrm{dsDNA}_{21}$ footprint), is disfavored, and duplexes are expected, on the contrary, to be rather scattered over the nanoparticle surfaces even for high DNA concentrations in the bulk. ${ }^{[17]}$

Direct SERS analysis was performed on the colloidal suspension by focusing a $532 \mathrm{~nm}$ laser with a long working distance lens so as to acquire averaged bulk SERS spectra with reproducible and well-defined spectral profiles. ${ }^{[11 b, 18]}$ Under this scenario - relatively small gaps and nanoparticle radius (i.e., large curvature at the interparticle junctions), combined with an excitation wavelength that matches the red-shifted gap plasmon resonances ${ }^{[18 a]}$ - the SERS intensity of aggregated colloidal systems is largely dominated by those few molecules located at the gap volume (hot spot). ${ }^{[12,19]}$ In support of this claim, we used an FDTD method to calculate the electromagnetic field distribution around a silver nanoparticle dimer in water (particle diameter $=22 \mathrm{~nm}$; gap distance $=2.5 \mathrm{~nm}$, such as that generated by a $\mathrm{dsDNA}_{21}$ duplex extended over the metallic surface) illuminated by a $532 \mathrm{~nm}$ laser. In Figure 2A, we show a horizontal and a vertical cut of the field intensity ( $x y$ - and $x z$-planes, respectively). We can appreciate that the EM field is strongly confined in the gap. Knowing how the electric field decays, we can estimate the Q7 volume of the hot spot considering the full width at half maximum (fwhm) of the electric intensity, $|E|^{2}$ (Figure 2B), in each direction. Approximating the hot-spot volume, $V$, as a cylinder whose height is given by the gap distance (i.e., $2.5 \mathrm{~nm}$ ) and the radius is $\approx 3 \mathrm{~nm}$ (i.e., fwhm), we can estimate $V=70 \mathrm{~nm}^{3}$. Based on the intrinsic size of the adsorbed dsDNA 21 surface complex $\left(\approx 30-35 \mathrm{~nm}^{3}\right)$ and the sparse surface coverage imposed by electrostatic repulsions between duplexes, we can safely estimate that no more than one $\mathrm{dsDNA}_{21}$ molecule is located at the hot spot. This means that one individual duplex placed at the gap is responsible for the vast majority of the SERS signal arising from the dimer. Such a conclusion can be qualitatively extended to the large ensembles of DNA-mediated clusters in suspension (i.e., the acquired SERS spectra specifically inform about the fraction of duplex molecules bridging cationic nanoparticles into aggregates, even for high DNA concentration).

\subsection{SERS Spectra Reveal DNA Strand Separation}

While AgSp colloids immediately aggregate upon addition of a short duplex, the complexation process between long genomic calf thymus DNA (ctDNA) and nanoparticles is slower and requires a longer time to reach its final equilibrium state. ${ }^{[6 c, 17]}$ For this reason, SERS analysis was performed on samples in the suspension after overnight incubation to reach binding equilibrium $^{[17]}$ (aggregates sitting at the bottom of the Eppendorf tube were resuspended just before the optical measurement). Salt concentration was maintained low (millimolar of $\mathbf{Q} 8$ $\mathrm{NaCl}$ ) throughout the whole study to minimize unspecific saltinduced aggregation of the nanoparticles (however, it is worth reminding that ion concentration at the nanoparticle surfaces 

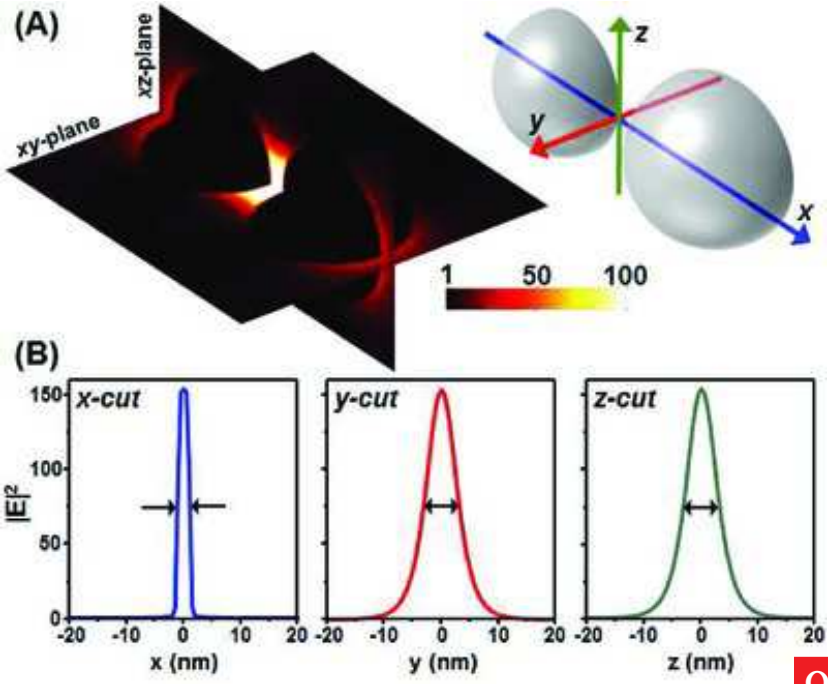

Figure 2. Electromagnetic near-field intensity calculations showing the volume size of the hot spot. A) The spatial near-field map of $|E|^{2}$ along the $x y$ - and $x z$-planes (black color represents a value of 0 while white represent a value of 100). B) The values of $|E|^{2}$ along each axis are also shown, where we can appreciate that the $x$-cut has the gap width while the field along the other two directions has a half-width at half-height around $3 \mathrm{~nm}$.

differs from the bulk $\left.{ }^{[20]}\right)$. Figure 3 illustrates the SERS spectra of ctDNA at a fixed concentration on AgSp colloids with different $\zeta$ potential and nanoparticle concentration. The spectra were normalized to the phosphate stretching band at $\approx 1089 \mathrm{~cm}^{-1}$, whose large insensitivity to changes in DNA structure makes it an ideal candidate for an internal standard.[11b-d,18a,21] The spectral profile of ctDNA undergoes a progressive reshaping upon addition to gradually diluted colloids. Among others, we highlight the drop in intensity of the guanine $(G)$ ring deformation band at $\approx 620 \mathrm{~cm}^{-1}$, the red-shifts of the ring-breathing modes of adenine $(\mathrm{A})\left(\approx 730 \mathrm{~cm}^{-1}\right)$ and cytosine $(\mathrm{C})+$ thymine $(\mathrm{T})$ $\left(785 \mathrm{~cm}^{-1}\right.$, main contribution from cytosine $\left.{ }^{[22]}\right)$, the intensity increase and large red-shift of the cytosine + ribose vibration at $\approx 1019 \mathrm{~cm}^{-1}$, and the remarkable weakening of the cytosine ring stretching at $\approx 1247 \mathrm{~cm}^{-1}$. All these changes are fully consistent with the DNA base unstacking and unpairing transition. ${ }^{[11 b, 18 a, 23]}$ Additionally, the broad composite carbonyl stretching band at $\approx 1651 \mathrm{~cm}^{-1}$ (mainly ascribed to thymine) suffers a large shift to lower frequency as also observed during thermal denaturation of B-form DNA. ${ }^{[21]}$ On the other hand, changes in $v(\mathrm{CO})$ band intensity are largely affected by the increasing relevance of the SERS background when the DNA signal drops as a result of the nanoparticle dilution and, thus, such spectral alterations cannot be reliably associated with perturbations of the DNA structure. Similar spectral changes are registered in the SERS spectra of the short dsDNA ${ }_{21}$ duplex (Figure S4, Supporting Information). Due to the particle size ( $\approx 22 \mathrm{~nm}$ diameter), the insertion of AgSp nanoparticles into the DNA grooves is hindered; ${ }^{[24]}$ thus, we can safely discard this process as a possible driving cause of the strand separation. On the contrary, minor spectral changes were observed when the colloidal supernatant was employed to similarly dilute AgSp (Figure S4, Supporting Information)

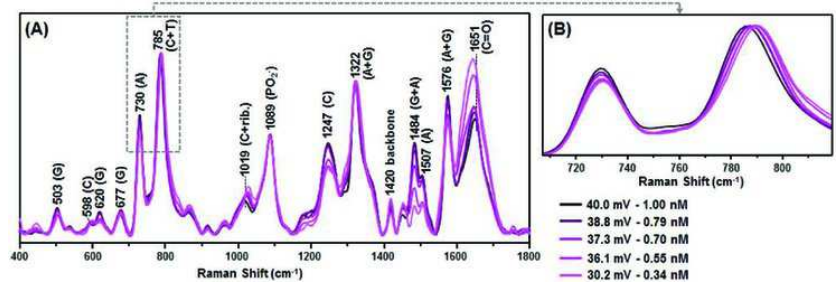

Figure 3. A) SERS spectra of ctDNA (final concentration: $7.5 \mu \mathrm{g} \mathrm{mL} \mathrm{L}^{-1}$ ) in AgSp colloids at different nanoparticle concentrations and $\zeta$ potential. B) Detail of the $710-820 \mathrm{~cm}^{-1}$ spectral region. The spectra were normalized to the phosphate band at $\approx 1089 \mathrm{~cm}^{-1}$. A general vibrational band assignment is also reported. ${ }^{[27],[45]} \mathrm{A}$, adenine; $\mathrm{C}$, cytosine; $\mathrm{T}$, thymine; $\mathbf{Q 1 0}$ $\mathrm{G}$, guanine; Rib., ribose; $\mathrm{PO}_{2}{ }^{-}$, phosphate group; and $(\mathrm{C}=\mathrm{O})$, carbonyl group.

demonstrating the key role of the surface charge in determining the extension of the duplex unzipping. It is also important to stress that the SERS profile of DNA-spermine complexes, formed by the interaction of the duplex with free spermine molecules in the bulk solution, differs drastically from that of DNA on AgSp colloids (Figure S5, Supporting Information), ${ }^{[15]}$ where the spermine molecules are restrained at the metal surface. This demonstrates that unbound spermine molecules in AgSp colloids do not play a significant role in shaping the final DNA SERS spectra, and, thus, the spectral changes illustrated in Figure 3 can be safely associated with strand separation. The SERS spectra shown in Figure 3 were obtained by adding a fixed amount of ctDNA to differently diluted AgSp colloids. This means that both surface charge (correlated with the $\zeta$ potential) and DNA/NP ratios, which affect the degree of nanoparticle clustering, were modified at the same time. To isolate these variables and separately investigate their role in the duplex unzipping, we performed two different SERS studies: in the first one, we progressively varied the DNA/NP ratio while keeping the colloidal $\zeta$ potential constant; in the second study, the effect of the nanoparticle surface charge on the spectral profile was monitored at fixed DNA/NP ratios. A short 21-mer single-stranded DNA (ssDNA $\left.{ }_{21}\right)$ was included as a probe molecule besides the long (ctDNA) and short $\left(\mathrm{dsDNA}_{21}\right)$ duplexes. Peak positions of the narrow and intense ring breathing bands at $\approx 730$ and $785 \mathrm{~cm}^{-1}$, ascribed to $\mathrm{A}$ and $\mathrm{C}+\mathrm{T}$ nucleobases, respectively, were selected as spectral markers of the state of the DNA tertiary structure (Figure 3B).

\subsection{The Role of Nanoparticle Clustering}

\subsubsection{SERS and SPR Analyses}

In Figure 4, we plotted the peak frequencies of the spectral markers observed at different DNA/NP ratios (milligrams of DNA per nanomoles of NPs, in logarithmic scale) for four sets of colloids at fixed surface charges ( $\zeta$ potential is equal to $\approx 40.0,38.6,33.2$, and $26.8 \mathrm{mV}$, respectively). The extension of the investigated ranges of DNA/NP ratios was defined by the acquisition of sufficiently intense and well-defined SERS spectra. At low DNA concentrations, we are mainly restricted by the overall number of scattering molecules in the sample, whereas in the presence of an excess of analyte, DNA saturation of the nanoparticle surfaces prevents the formation of efficient hot spots (i.e., small 


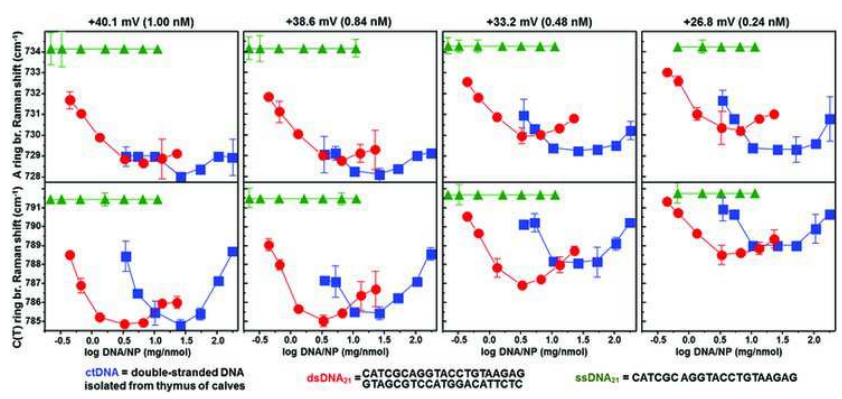

Figure 4. Frequency shifts of adenine and cytosine (+thymine) ring breathing bands for SERS spectra of ctDNA, dsDNA ${ }_{21}$, and ssDNA 21 acquired in AgSp colloids at fixed $\zeta$ potentials. For each batch of colloids, the DNA concentration, expressed as milligrams of analyte per nanomoles of nanoparticles (logarithmic scale), was progressively increased up to values that still yield detectable signals. Error bars equal to two standard deviations $(N=3)$.

number of hot spots in the sample). The results show that no spectral shifts were observed for ssDNA 21 , whereas both short and genomic duplexes reveal notable changes in their spectral profiles (Figure S6, Supporting Information), with the appearance of a frequency minimum at intermediate DNA/NP ratios, corresponding to the minimal perturbation of the double-helix stability by cationic nanoparticles of a specific $\zeta$ potential value. Overall, the position of such frequency minima moves to larger Raman shifts when the nanoparticle surface charge is decreased. It is also worth noting that the peak frequencies of both $\mathrm{A}$ and $\mathrm{C}+\mathrm{T}$ ring breathing bands consistently remain far below than those of $\mathrm{ssDNA}_{21}$, except when very low DNA/NP ratios are investigated in colloids with lowest surface charge (Figure 4, AgSp with a $\zeta$ potential of $+26.8 \mathrm{mV}$ ). These general observations are valid for both short and genomic DNA duplexes. On the contrary, for AgSp colloids at a given $\zeta$ potential, we observe that minimal duplex separation (corresponding to the most blue-shifted ring breathing bands) always takes place at lower DNA/NP ratios for $\mathrm{dsDNA}_{21}$ than ctDNA (Figure 4). Besides the obvious difference in length, dsDNA 21 and ctDNA deviate in nucleobase sequence and composition (GC content of $\approx 52.4 \%$ for short and $\approx 41.9 \%$ for genomic DNAs). To examine the role of these latter variables, we performed the identical spectral analysis described in Figure 4 but for two 22-bp duplexes either composed of CG or AT nucleobases (dsCG and dsAT, respectively; see Figure S7 in the Supporting Information). Overall, the trends of peak frequency shifts for dsCG and dsAT largely match those of dsDNA dFig- $^{21}$ (Fig ure S7, Supporting Information) indicating that, rather than base sequence and composition, the strand length parameter is likely to play the major role in determining the marked offset in double-strand unzipping for dsDNA $\mathrm{A}_{21}$ and ctDNA at different DNA/NP ratios (Figure 4). Given these results, questions arise as to which mechanisms lie behind the connection between DNA/NP ratio factor and the characteristic changes in the tertiary structure of DNA duplexes electrostatically trapped at the interparticle junctions. To gain insights on this intriguing issue, we have undertaken detailed studies on the evolution of the optical properties of nanoparticle clusters in suspension as well as performed MD simulations on model DNA-mediated clusters.
In addition to the large SERS enhancements localized at the interparticle gaps, the plasmonic coupling of interacting silver nanoparticles is responsible for the reshaping of the colloidal extinction profile with the appearance of new gap plasmon resonances red-shifted with respect to the LSPR. ${ }^{[25]}$ However, the SERS enhancement distribution and far-field SPR responses from large ensembles of nanoparticle clusters have different spatial averaging properties due to the different spatial localization of the plasmon resonances. ${ }^{[26]}$ Thus, UV/vis/NIR spectroscopy analysis of DNA-mediated aggregates can provide important complementary information on the correlation between strand separation, as revealed by SERS, and general geometrical features of nanoparticle clusters (i.e., gaps separation, size, and shape).$^{[12,25]}$ Figure 5A displays the extinction spectra of AgSp colloids $\left([\mathrm{NP}] \approx 1.00 \times 10^{-9} \mathrm{M} ; \zeta\right.$ potential $\left.\approx 40 \mathrm{mV}\right)$ registered $60 \mathrm{~min}$ (red line) or $1 \mathrm{~d}$ (pink line) after the addition of increasing amounts of dsDNA 21 . At low DNA/NP ratios $(0.64$ $\mathrm{mg} \mathrm{nmol}{ }^{-1}$ ), we observe a very mild nanoparticle aggregation after $60 \mathrm{~min}$, consistently with the small number of duplexes per nanoparticle that limits the extensive formation of DNAmediated clusters. However, the perturbation introduced in the colloidal system eventually triggers further aggregation events that yield larger features with broader and weaker plasmonic contributions. ${ }^{[26 \mathrm{~b}]}$ In fact, after $1 \mathrm{~d}$, the particles undergo an irreversible coalescence which also leads to the visible deposition of films of silver nanoparticles at the Eppendorf tube surfaces. Differently, for higher dsDNA 21 concentrations, the colloidal system rapidly aggregates into stable clusters that can be easily redispersed after sedimentation. Notably, when DNA concentration is further increased as much as to start saturating metallic surfaces, the extent of interparticle coupling decreases as revealed by the overall blue-shift of the gap plasmon resonances for DNA/NP ratio of $12.8 \mathrm{mg} \mathrm{nmol}^{-1}$ as compared to $3.2 \mathrm{mg}$ $\mathrm{nmol}^{-1}$ (Figure 5A).

The absorbance ratio at 700 and $391 \mathrm{~nm}\left(I_{700} / I_{\text {LSPR }}\right)$ was selected to better visualize the effect of time and DNA/NP ratio on the ensemble of gap-plasmon resonances (Figure 5B). Here, we can observe that, for DNA/NP ratios below $\approx 2 \mathrm{mg} \mathrm{nmol}^{-1}(\approx 0.3$ in the logarithmic scale), the duplex coverage of the silver surface is not sufficient to either induce extensive nanoparticle aggregation or impart long-term colloidal stability. In this regard, SERS analysis was also performed on each sample at $10 \mathrm{~min}, 60 \mathrm{~min}$, and $1 \mathrm{~d}$, showing neither appreciable peak shifts of the spectral markers nor significant changes in absolute intensity over time. This suggests that the unspecific colloidal aggregation that follows the initial DNA-mediated cluster formation does not affect in a tangible way the final SERS profile. On the other hand, for DNA/NP ratios above $\approx 3 \mathrm{mg} \mathrm{nmol}^{-1}(\approx 0.5$ in the logarithmic scale), we observe the rapid formation of stable clusters in suspension. The extent of respective plasmon coupling appears to gradually decrease as the DNA/NP ratio is progressively raised (up to the largest DNA/NP value presented in this study).

The $60 \mathrm{~min}$ aged mixtures were also analyzed by dynamic light scattering (DLS) to extract the corresponding hydrodynamic size and size distribution of the DNA-mediated aggregates (Figure S8A, Supporting Information). These samples were selected as they provide a close picture of the colloidal system at the equilibrium while largely avoiding the unspecific colloidal aggregation observed for low DNA/NP ratio at longer 

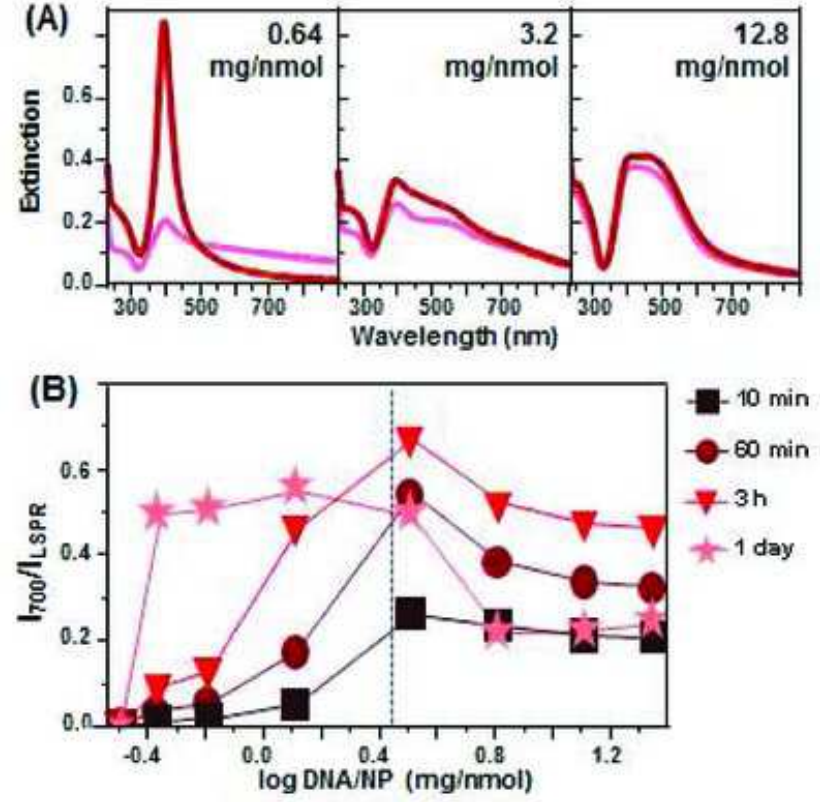

Figure 5. A) Extinction spectra of AgSp colloids ([NP] $\approx 1.00 \times 10^{-9}$ $\mathrm{M}, \zeta$ potential $\approx 40 \mathrm{mV}$ ) after the addition of different amounts of analyte, corresponding to $\approx 0.64,3.2$, and $12.8 \mathrm{mg}$ of dsDNA 21 per nmol of nanoparticles. The spectra were acquired $60 \mathrm{~min}$ and $1 \mathrm{~d}$ after the addition of dsDNA $A_{21}$ to the colloids (red and pink curves, respectively). B) Ratio of the extinction intensities at $700 \mathrm{~nm}$ over the intensity at the LSPR maximum $\left(I_{700} / I_{L S P R}\right)$ against DNA/NP ratios ( $\mathrm{mg}$ of $\mathrm{ds} \mathrm{DNA}_{21}$ per $\mathrm{nmol}$ of NP, in logarithmic scale). The intensity ratios were recorded at different times after the addition of the dsDNA ${ }_{21}$ in the as-synthesized AgSp colloids. Aggregates sitting at the bottom of the Eppendorf tubes were briefly resuspended just before the optical measurement. The blue dotted lines broadly separate the lower and upper DNA/NP ratio ranges corresponding to the formation of unstable and stable clusters in suspension, respectively.

Q11

time. Differently to what observed for the extinction ratio, the average hydrodynamic diameter of the aggregates shows a continuous increment with the increasing of the DNA content. On a purely qualitative basis, such a trend can also be captured in scanning electron microscopy (SEM) images of spin-coated

Q12 samples (Figure S8B-D, Supporting Information). This result directly entails that the decrease of the $I_{700} / I_{\text {LSPR }}$ value observed for DNA/NP ratios larger than $\approx 3 \mathrm{mg} \mathrm{nmol}^{-1}$ cannot be ascribed to a reduction of the cluster size but to an enlargement of the average gaps within nanoparticle aggregates. Here, when a large number of DNA molecules compete for adhesion on a few surface sites, the surface crowding and the electrostatic repulsion between neighboring duplexes hamper the necessary conformational changes required to maximize the number of contacting points with the surface. ${ }^{[17]}$ Thus, we can reasonably expect that, instead of homogeneously extending over the metallic surface, DNA molecules bind the silver surface adopting a broad set of tilted conformations, which lead to larger interparticle separations in aggregates. This picture is fully consistent with previous molecular dynamic simulations that described the DNA adsorption on a spermine-bound silver surface as a multistep process. ${ }^{[11 c]}$ According to this mechanism, after a fast recog- nition phase, the total adhesion of short DNA strands is achieved by conformational changes triggered by electrostatic interactions with spermine molecules adsorbed on the surface. This second step can be hindered by an overcrowded silver surface and/or without the necessary amount of free spermine molecules, thus leading to aggregates characterized by properly adsorbed duplexes coexisting with partly bound DNA molecules.

The evolution of the DNA-driven nanoparticle clusterization was also investigated for ctDNA (Figure S9, Supporting Information). The semiquantitative analysis of the extinction spectra did reveal minimal interparticle plasmonic coupling at very low or very high ctDNA concentrations, but it was not possible to discern any further distinctive pattern. Most likely, this is the consequence of the coexistence of many additional aggregation routes. Opposite to what happens for the adsorption of $\approx 7 \mathrm{~nm}$ rod-like duplexes on AgSp nanoparticles, in the case of long genomic DNA, the silver particles are the nanoscale objects that accumulate at the duplex chain, leading to the progressive compaction of the elongated coil which can also involve the wrapping of the chain around individual nanospheres. ${ }^{[27]}$ Such a compaction process consists of several intermediate states with uneven distributions of nanoparticles along the chain. ${ }^{[27]}$ Furthermore, nanoparticles in suspension tend to agglomerate at the surfaces of other nanoparticles previously coordinated to the genomic strand..$^{[27 a]}$

The antithetical role played by DNA of different chain lengths on the nanoparticle aggregation pattern (i.e., nanoparticles collect short dsDNA ${ }_{21}$ at their surfaces versus long genomic ctDNA collects nanoparticles at the duplex chain) is also well reflected in the UV-vis analysis of the colloidal supernatants upon removal of DNA-mediated clusters (Figure S10, Supporting Information). Monitoring of the optical absorption of DNA at $260 \mathrm{~nm}$ reveals that a progressively higher concentration of unbound $\mathrm{dsDNA}_{21}$ is present in the supernatant for DNA/NP ratio above $\approx 3 \mathrm{mg} \mathrm{nmol}^{-1}(\approx 0.5$ in the logarithmic scale; see Figure S10C in the Supporting Information), which precisely corresponds to the stability threshold for dsDNA 21 -driven nanoparticle assemblies (i.e., when the metallic surfaces are largely saturated with short duplexes that act in this situation also as stabilizing ligands). Notably, unbound dsDNA 21 in the supernatant are observed for AgSp colloids with higher $\zeta$ potential, which indicates that duplex surface density increases with the nanoparticle surface charge. On the other hand, in the case of ctDNA, residual duplexes in the supernatant are only detected for the largest DNA/NP ratio (Figure S10D, Supporting Information) together with a weak additional band at $\approx 398 \mathrm{~nm}$ ascribable to very sparse individual nanoparticles attached to the chain. For all ctDNA/NP ratios below that value, the relatively high weight of the ctDNAnanoparticle complexes leads to sedimentation.

\subsubsection{Molecular Dynamic Simulations}

The illustrated SERS and far-field SPR data are the highly averaged responses of very complex samples containing a large number of polydispersed aggregates. Modeling systems of such sizes and complexity are, at present, unfeasible. Nonetheless, MD-based simulations of model DNA-mediated clusters may give a hint of the potential connection between the cluster geometrical features and the perturbation of the interacting DNA at 
the junctions. In particular, two model systems were envisioned, a dimer (2NP) and a trimer (3NP) with a compact geometry (see Figure 6 and Figure S11A, Supporting Information), as the simplest prototypes of small and larger aggregates, respectively. Both models included a single dsDNA ${ }_{21}$ molecule adopting an extended conformation at the interface of each gap. In Figure 6 , we show the free energy surfaces calculated as a function of the separation between nanoparticles and the average distance between base pairs belonging to different DNA strands. In both 2NP and 3NP systems, the deepest minima corresponded to states showing the double helix of DNA in the B-form. Specifically, in the 2NP cluster model, the global minimum was found at a relatively large interparticle distance of about $29 \AA$ (Figure 6A). Conversely, in the trimer model, the most stable state was located at much narrower separations (24-25 A, Figure 6B). This finding clearly indicates a trend, whereby smaller aggregates tend to stretch or, at least, to easily interconvert between states of different contractions, while more compact clusters are highly preferred in the case of larger aggregates. This effect can be explained through a DNA-mediated binding cooperativity between the three nanoparticles, which is missing in the 2NP model. Most importantly, however, is that the investigated systems show a remarkably different behavior along the reaction coordinate describing the separation between the two strands of the duplex. Indeed, an incipient unzipping could be observed in the 2NP system, as it can be assessed by the multiple minima located in the free energy surface at strand separations greater than $5 \AA$. This behavior is only marginally found in the 3NP model, most likely as a consequence of the cluster aggregate compaction that hinders the double strand unzipping. Taken as a whole, the outcome of MD simulations suggests that the different sizes and geometrical features of DNA-mediated clusters play indeed an important role in determining the structural features of the duplex at the gaps. This provides a new tool for interpreting the experimental trends as illustrated in Figures 4 and 5. On these bases, we propose the following. Minimal strand separation is fulfilled at intermediate DNA/NP values when cluster aggregate compaction is maximized. However, when DNA concentration is low, molecules extend homogenously over the metallic surface, and cluster compaction is improved only by increasing the number of nanoparticles per aggregate. On the other hand, for large DNA/NP ratios, cluster compaction diminishes as a result of the increased separation between nanoparticles imposed by reorientation of the DNA molecules over the silver surfaces.

\subsection{The Role of Nanoparticle Surface Charge}

Based on the outcome of the data illustrated in Figure 4, we subsequently fixed the DNA/NP ratios at those values associated with minimal duplex perturbation $\left(\approx 19 \mathrm{mg} \mathrm{nmol}^{-1}\right.$ for ctDNA and $3.2 \mathrm{mg} \mathrm{nmol}^{-1}$ for dsDNA ${ }_{21}$ ) while monitoring the $\mathrm{C}+\mathrm{T}$ ring breathing peak position on nanoparticles at decreasing surface charge (Figure 7). In this case, short and genomic duplexes share the same qualitative behavior, presenting a jump in peak frequency for $\zeta$ potential values in the $\approx 30-35 \mathrm{mV}$ range. An identical result was observed for the A spectral marker (Figure S12, Supporting Information).

The same study was further extended to a 21-bp RNA duplex (dsRNA $\left.{ }_{21}\right)$ which, in addition to nucleobase sequence and com-

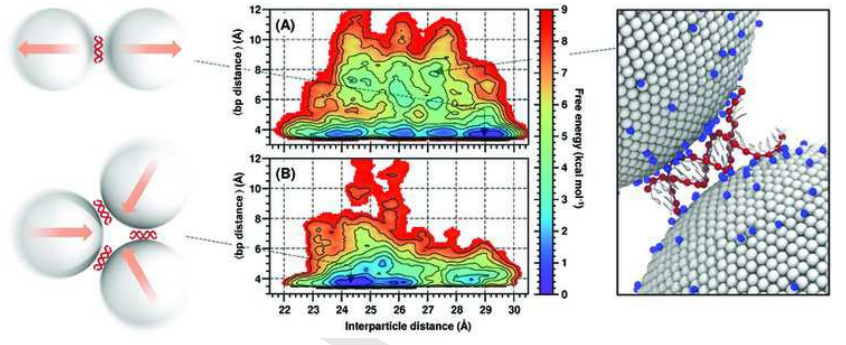

Figure 6. A,B) Free energy surfaces computed as a function of the interparticle distance and the average distance between complementary base pairs for 2 NP and 3 NP model systems. Iso-lines are plotted with a step of $1 \mathrm{kcal} \mathrm{mol}^{-1}$. Pictorial representations of the mechanical features of the cluster aggregates, and a representative configuration showing partial DNA unzipping extracted from MD-based simulations, are also shown (left and right panels, respectively).

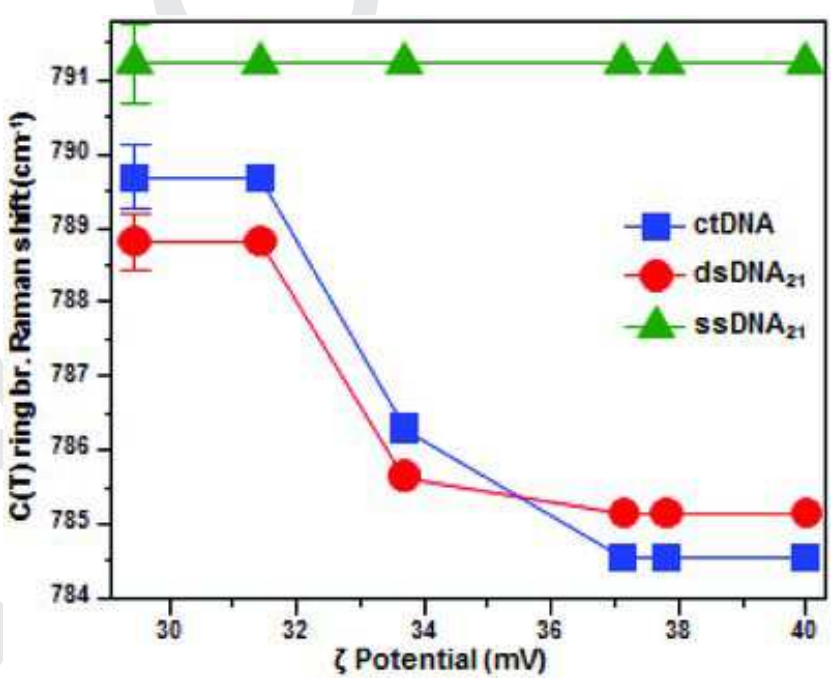

Figure 7. Frequency shift of cytosine (+thymine) ring breathing bands for SERS spectra of ctDNA, dsDNA 21 , and ssDNA 21 acquired in AgSp colloids at different $\zeta$ potentials upon dilution with Milli-Q water. DNA/NP ratio was kept constant to $19 \mathrm{mg} \mathrm{nmol}{ }^{-1}$ for ctDNA, $3.2 \mathrm{mg} \mathrm{nmol}^{-1}$ for $\mathrm{dsDNA}_{21}$, and $1.6 \mathrm{mg} \mathrm{nmol}^{-1}$ for ssDNA 21 . Error bars equal to two standard deviations $(N=4)$.

position, differs from dsDNA also by the helix geometry (mainly the B-form for dsDNA 21 and A-form for dsRNA 21 ) and the sugar molecular structure. ${ }^{[18 a]}$ Despite the structural differences, our SERS data indicate that the spectral shift dependency of dsRNA 21 from the colloidal surface charge is qualitatively analog to those of short and long DNA duplexes (Figure S12, Supporting Information). Thus, when the cluster geometry variable, which is strictly correlated to the chain length, is removed by fixing the DNA/NP ratios at the minimal double-helix perturbation, the differences of spectral behavior for short and genomic duplexes are largely levelled out. A possible explanation may be found in the electrostatic nature of the DNA/NP interaction. dsDNA initially adsorbs onto a nanoparticle, then screens its positive charge thereby favoring the subsequent approaching of a second nanoparticle to generate the corresponding interparticle junction. As previously simulated by means of MD methods, ${ }^{[11 c]}$ the 
duplex binding onto the first nanoparticle occurs on the nanosecond time scale, where the flexibility of the surface spermine molecules contributes to promoting the optimum interaction with the phosphate groups aligned along the DNA backbone. On the other hand, the second approaching nanoparticle "sees" a duplex whose degrees of freedom are largely reduced due to the interaction with the solid phase. Thus, we may speculate that such a second DNA-NP interaction could occur to the detriment of the duplex stability, especially when the spermine surface density is decreased below a critical level (i.e., when the $\zeta$ potential is lowered). Under this scenario, no significant differences are expected to be revealed by the SERS signal of duplexes with different lengths, since their SERS spectra largely result from the contribution of chain fragments with similar size (limited by the hot spot volume) and carrying equally spaced phosphate groups on the DNA backbone.

\section{Conclusion}

In summary, the outcomes of the SERS analysis can be summarized as follows. Overall, the results indicate that the interaction of the cationic nanoparticles with double-stranded DNA leads to (at least partial) strand separation of dsDNA population that molecularly mediates the nanoparticle clustering (i.e., DNA molecules at the interparticle junctions). The extent of such structural deformation is highly dependent on (i) the DNA/NP ratio and (ii) the nanoparticle surface charge. The former parameter directly affects the aggregation profile which is, in turn, strictly related to the length of the duplex (whereas base composition did not appear here to play a relevant role). Regardless of the chain length, it was possible to recognize a qualitatively common trend where unzipping of the double helixes is progressively maximized at high and low DNA/NP ratios, for a given $\zeta$ potential. The potential correlation between the aggregation features of a DNA-mediated cluster and the tendency of the DNA molecule trapped at the interparticle gap to undergo unzipping was further investigated and confirmed by molecular dynamics in combination with additional SPR analysis. Differently, once fixed the DNA/NP ratio (i.e., the degree of aggregation) to a value corresponding to the minimal duplex perturbation and monitored SERS spectra for different nanoparticle surface charges, we observe a sudden transition from a dominant duplex conformation to mainly separated strands below a critical $\zeta$ potential threshold. This transition appears to be largely independent not only of nucleobase composition but also of the chain length and double helical geometry. This extensive work provides new fundamental insights into the interaction between nucleic acids and nanoparticles, and how the integrity of the double-helical structure is perturbed by the cooperative binding of nano-objects.

\section{Experimental Section}

Materials: All reagents were of analytical grade and used as received. All chemicals were obtained from Sigma-Aldrich except for short ssDNA and ssRNA oligonucleotides (Table S1, Sup- porting Information), which were purchased from Eurofins Genomics.

Cationic Silver Nanoparticles: Synthesis of positively charged colloids was carried as previously described. ${ }^{[18 a]} \zeta$ potential measurements were performed by diluting the nanoparticles with either the colloidal supernatant or Milli-Q water. The supernatant was obtained by removing the nanoparticles from colloids via centrifugation at $13400 \mathrm{rpm}$ for $40 \mathrm{~min}$.

SERS Measurements: SERS spectra at a fixed ctDNA concentration in differently diluted AgSp colloids (Figure 3) were obtained by adding $10.2 \mu \mathrm{L}$ of ctDNA $117.2 \mathrm{mg} \mathrm{L}^{-1}$ to $150 \mu \mathrm{L}$ of colloids. The data illustrated in Figure 4 and Figure S7 (Supporting Information) were extracted from the SERS spectra of ctDNA $\mathrm{A}_{21}$ and short ss- and ds-oligonucleotides (ssDNA 21 , dsDNA ${ }_{21}, \mathrm{dsCG}$, and dsAT) for different DNA/NP ratios (and fixed AgSp $\zeta$ potential). DNA/NP ratio $(R)$ was expressed in milligrams of DNA per nanomoles of NP. In each case, the ratios were expressed as follows: for ctDNA $21, R=3.6,5.4,10.8,27,54,107.9$, and $186 \mathrm{mg}$ $\mathrm{nmol}^{-1}$; for ssDNA $21, R=0.21,0.32,0.64,1.61,3.21,6.43$, and $11.10 \mathrm{mg} \mathrm{nmol}^{-1}$; and finally for dsDNA $21, R=0.43,0.64,1.29$, $3.21,6.43,12.85$, and $22.16 \mathrm{mg} \mathrm{nmol}^{-1}$ (identical values were used for dsCG and dsAT analyses). These samples were pre- Q13 pared by adding different aliquots of DNA solutions, obtained upon proper dilution of the stock solutions, to $150 \mu \mathrm{L}$ of colloids. The volume of such aliquots ranged between 3 and $10 \mu \mathrm{L}$ in order to avoid significant alteration of the final nanoparticle concentration. Data reported in Figure 7 and Figure S12 (Supporting Information) were extracted from SERS spectra of DNA/RNA in AgSp at different $\zeta$ potentials and fixed DNA/NP ratios $(R$ $=19 \mathrm{mg} \mathrm{nmol}^{-1}$ for ctDNA, $3.2 \mathrm{mg} \mathrm{nmol}^{-1}$ for dsDNA 21 and $\mathrm{dsRNA}_{21}$, and $1.6 \mathrm{mg} \mathrm{nmol}^{-1}$ for ssDNA ${ }_{21}$ ). These samples were also prepared according to the previously described procedure. All samples were prepared and left to equilibrate overnight at $4{ }^{\circ} \mathrm{C}$. The samples were quickly redispersed before the SERS measurements.

$\zeta$ Potential and DLS Measurements: $\zeta$ potential and DLS measurements were performed on a Malvern Nano Zetasizer NanoZS (Malvern Instruments Inc.,UK) equipped with a $4 \mathrm{~mW} \mathrm{He}-$ $\mathrm{Ne} 633 \mathrm{~nm}$ laser. $\zeta$ potential measurements were acquired under the Smoluchowski model, used for most aqueous solutions. All samples were measured by triplicate at $25^{\circ} \mathrm{C}$ and constant $\mathrm{pH}$ (6.2). A universal dip cell (containing the electrodes) was inserted into the samples, previously placed in disposable PS cuvettes. Q14 Hydrodynamic size measurements were acquired with a $173^{\circ}$ backscattered angle and calculated via the Stokes-Einstein equation. In this case, all samples were diluted six times in Milli-Q water, in order to avoid issues related to absorption and multiple scattering events from the sample. As for $\zeta$ potential measurements, the samples were measured by triplicate at $25^{\circ} \mathrm{C}$, using disposable cuvettes.

Equipment and Instrument Settings: A Thermo Scientific Evolution $201 \mathrm{UV}$-visible spectrophotometer was employed to acquire UV-vis spectra. $\zeta$ potential and DLS measurements were performed on a Malvern Nano Zetasizer. SEM images were acquired with an environmental scanning electron microscope (JEOL 6400). SERS experiments were performed with a Renishaw InVia Reflex confocal microscope equipped with a highresolution grating consisting of 1800 grooves $\mathrm{mm}^{-1}$ for visible wavelengths, additional bandpass filters, and a CCD camera. A 
long working distance objective (0.17 NA, working distance 30 $\mathrm{mm}$ ) was used to focus a $532 \mathrm{~nm}$ laser onto the colloidal solutions. SERS spectra were acquired at room temperature with 20 accumulations, $15 \mathrm{~s}$ exposure time, and a laser power at the sample of $6.9 \mathrm{~mW}$. All SERS spectra illustrated in the manuscript were obtained by averaging the three different replications (per each sample). DLS samples were prepared by diluting six times the SERS samples in Milli-Q water prior to the measurements.

Molecular Dynamics Simulations: Molecular dynamics simulations were performed on two DNA-mediated cluster models, hereafter referred to as $2 \mathrm{NP}$ and $3 \mathrm{NP}$. The systems contained two and three positively charged nanoparticles, respectively. A model of dsDNA 21 was placed between each nanoparticle pair to simulate a stationary cluster aggregate. Further details are included in the Supporting Information.

\section{Supporting Information}

Supporting Information is available from the Wiley Online Library or from the author.

\section{Acknowledgements}

The work was funded by Ministerio de Economia y Competitividad (CTQ2014-59808R), European Research Council (PrioSERS FP7/2014 623527), Generalitat de Catalunya (2014SGR-480), and Secretaria d'Universitats i Recerca del Departament d'Economia i Coneixement de la Generalitat de Catalunya.

\section{Conflict of Interest}

The authors declare no conflict of interest.

\section{Keywords}

nanoparticles, nucleic acids, strand separation, surfaceenhanced Raman

Received: June 20, 2017 Published Online: MM DD, YYYY

[1] a) A. Samanta, I. L. Medintz, Nanoscale 2016, 8, 9037; b) S. J. Tan, M. J. Campolongo, D. Luo, W. L. Cheng, Nat. Nanotechnol. 2011, 6, 268; c) G. H. Clever, M. Shionoya, Coord. Chem. Rev. 2010, 254, 2391; d) M. R. Jones, N. C. Seeman, C. A. Mirkin, Science 2015, 347, 12.

[2] a) J. I. Cutler, E. Auyeung, C. A. Mirkin, J. Am. Chem. Soc. 2012, 134, 1376 ; b) P. S. Randeria, M. R. Jones, K. L. Kohlstedt, R. J. Banga, M. Olvera De La Cruz, G. C. Schatz, C. A. Mirkin, J. Am. Chem. Soc. 2015, 137, 3486; c) S. Ohta, D. Glancy, W. C. W. Chan, Science 2016, $351,841$.

[3] J. M. Carnerero, A. Jimenez-Ruiz, P. M. Castillo, R. Prado-Gotor, ChemPhysChem 2017, 18, 17.
[4] a) S. T. Kim, K. Saha, C. Kim, V. M. Rotello, Acc. Chem. Res. 2013, 46, 681; b) A. Chompoosor, K. Saha, P. S. Ghosh, D. J. Macarthy, O. R. Miranda, Z. J. Zhu, K. F. Arcaro, V. M. Rotello, Small 2010, 6, 2246; c) R. D. Handy, F. Von Der Kammer, J. R. Lead, M. Hassellöv, R. Owen, M. Crane, Ecotoxicology 2008, 17, 287; d) F. Paillusson, V. Dahirel, M. Jardat, J.-M. Victor, M. Barbi, Phys. Chem. Chem. Phys. 2011, 13, 12603; e) Y. Liu, W. Li, F. Lao, Y. Liu, L. Wang, R. Bai, Y. Zhao, C. Chen, Biomaterials 2011, 32, 8291; f) G. Han, C. T. Martin, V. M. Rotello, Chem. Biol. Drug Des. 2006, 67, 78; g) J. A. Nash, A. L. Kwansa, J. S. Peerless, H. S. Kim, Y. G. Yingling, Bioconjugate Chem. 2017, 28, 3.

[5] a) C. M. McIntosh, E. A. Esposito III, A. K. Boal, J. M. Simard, C. T. Martin, V. M. Rotello, J. Am. Chem. Soc. 2001, 123, 7626; b) B. Kang, M. A. Mackey, M. A. El-Sayed, J. Am. Chem. Soc. 2010, 132, 1517; c) N. M. Schaeublin, L. K. Braydich-Stolle, A. M. Schrand, J. M. Miller, J. Hutchison, J. J. Schlager, S. M. Hussain, Nanoscale 2011, 3,410 .

[6] a) H. Kamata, A. Zinchenko, S. Murata, Colloid Polym. Sci. 2011 289, 1329; b) J. G. Railsback, A. Singh, R. C. Pearce, T. E. McKnight, R. Collazo, Z. Sitar, Y. G. Yingling, A. V. Melechko, Adv. Mater. 2012 24, 4261; c) S. C. Bera, K. Sanyal, D. Senapati, P. P. Mishra, J. Phys. Chem. B 2016, 120, 4213 .

[7] L. R. Ferguson, W. A. Denny, Mutat. Res., Fundam. Mol. Mech. Mutagen. 2007, 623, 14.

[8] K. Aslan, I. Gryczynski, J. Malicka, E. Matveeva, J. R. Lakowicz, C. D. Geddes, Curr. Opin. Biotechnol. 2005, 16, 55.

[9] S. Chen, Z. Cao, S. Jiang, Biomaterials 2009, 30, 5892.

[10] J. A. Nash, A. Singh, N. K. Li, Y. G. Yingling, ACS Nano 2015, 9 12374.

[11] a) S. Schlücker, Angew. Chem., Int. Ed. 2014, 53, 4756; b) L. Guerrini, Ž. Krpetić, D. van Lierop, R. A. Alvarez-Puebla, D. Graham, Angew. Chem., Int. Ed. 2015, 54, 1144; c) M. Masetti, H.-n. Xie, Ž. Krpetić, M. Recanatini, R. A. Alvarez-Puebla, L. Guerrini, J. Am. Chem. Soc 2015, 137, 469; d) L.-J. Xu, Z.-C. Lei, J. Li, C. Zong, C. J. Yang, B. Ren, J. Am. Chem. Soc. 2015, 137, 5149; e) S. R. Panikkanvalappil, S. M. Hira, M. A. Mahmoud, M. A. El-Sayed, J. Am. Chem. Soc. 2014, 136, 15961; f) S. R. Panikkanvalappil, M. A. Mahmoud, M. A. Mackey, M. A. El-Sayed, ACS Nano 2013, 7, 7524; g) J. MorlaFolch, P. Gisbert-Quilis, M. Masetti, E. Garcia-Rico, R. A. AlvarezPuebla, L. Guerrini, Angew. Chem., Int. Ed. 2017, 56, 2381; h) J. Wang, K. Koo, E. J. H. Wee, Y. Wang, M. Trau, Nanoscale 2017 https://doi.org/10.1039/C6NR09928A.

[12] E. C. Le Ru, P. G. Etchegoin, Principles of Surface-Enhanced Raman Spectroscopy, 2009.

[13] M. A. Brown, A. Goel, Z. Abbas, Angew. Chem., Int. Ed. 2016, 55, 3790.

[14] a) J. Yguerabide, E. E. Yguerabide, Anal. Biochem. 1998, 262, 137. b) J. Yguerabide, E. E. Yguerabide, Anal. Biochem. 1998, 262, 157.

[15] A. Torres-Nunez, K. Faulds, D. Graham, R. A. Alvarez-Puebla, L. Guerrini, Analyst 2016, 141, 5170.

[16] A. Estevez-Torres, D. Baigl, Soft Matter 2011, 7, 6746.

[17] X. Zhang, M. R. Servos, J. Liu, Langmuir 2012, 28, 3896.

[18] a) J. Morla-Folch, H.-n. Xie, R. A. Alvarez-Puebla, L. Guerrini, ACS Nano 2016, 10, 2834; b) R. Aroca, Surface-Enhanced Vibrational Spectroscopy, John Wiley \& Sons, Chichester, UK 2006.

[19] a) S. L. Kleinman, B. Sharma, M. G. Blaber, A. I. Henry, N. Valley, R. G. Freeman, M. J. Natan, G. C. Schatz, R. P. Van Duyne, J. Am. Chem Soc. 2013, 135, 301; b) E. C. Le Ru, P. G. Etchegoin, M. Meyer, J. Chem. Phys. 2006, 125, 204701.

[20] C. Pfeiffer, C. Rehbock, D. Hühn, C. Carrillo-Carrion, D. J. de Aberasturi, V. Merk, S. Barcikowski, W. J. Parak, J. R. Soc., Interface 2014 11.

[21] J. G. Duguid, V. A. Bloomfield, J. M. Benevides, G. J. Thomas, Biophys. J. 1996, 71, 3350 . 
[22] J. Morla-Folch, H.-n. Xie, P. Gisbert-Quilis, S. Gómez-de Pedro, N. Pazos-Perez, R. A. Alvarez-Puebla, L. Guerrini, Angew. Chem., Int. Ed. 2015, 54, 13650.

[23] L. Movileanu, J. M. Benevides, G. J. Thomas, Biopolymers 2002, 63, 181.

[24] a) P. Rivera Gil, D. Hühn, L. L. del Mercato, D. Sasse, W. J. Parak, Pharmacol. Res. 2010, 62, 115; b) Y. Pan, S. Neuss, A. Leifert, M. Fischler, F. Wen, U. Simon, G. Schmid, W. Brandau, W. JahnenDechent, Small 2007, 3, 1941.

[25] V. Giannini, A. I. Fernandez-Dominguez, S. C. Heck, S. A. Maier, Chem. Rev. 2011, 111, 3888.

[26] a) E. C. Le Ru, C. Galloway, P. G. Etchegoin, Phys. Chem. Chem. Phys. 2006, 8, 3083; b) L. Guerrini, F. McKenzie, A. W. Wark, K. Faulds, D. Graham, Chem. Sci. 2012, 3, 2262.

[27] a) A. A. Zinchenko, T. Sakaue, S. Araki, K. Yoshikawa, D. Baigl, J. Phys. Chem. B 2007, 111, 3019; b) A. A. Zinchenko, K. Yoshikawa, D. Baigl, Phys. Rev. Lett. 2005, 95, 228101. 
Q1 APT to AU: Please provide the department/institution names in affiliations 4 and 5.

Q2 APT to AU: Please provide the highest academic title (either Dr. or Prof.) for all authors, where applicable.

Q3 APT to AU: Please check that the edit to the preceding sentence is OK and that the meaning has not been changed.

Q4 APT to AU: Please define 'MS' at the first occurrence in the text.

Q5 APT to AU: Please define 'TEM' at the first occurrence in the text.

Q6 APT to AU: Please define 'PBS' at the first occurrence in the text.

Q7 APT to AU: Please define 'EM' at the first occurrence in the text.

Q8 APT to AU: Please check that the edits to the preceding sentence are OK and that the meaning has not been changed. Kindly also check similar change elsewhere in the text.

Q9 APT to AU: Please check that the edits to the preceding sentence are OK and that the meaning has not been changed.

Q10 APT to AU: Reference (45) has been cited in the caption to Figure 3 but is not present in the reference list. Please check.

Q11 APT to AU: Please check that the edit to the preceding sentence is OK and that the meaning has not been changed.

Q12 APT to AU: Please confirm if 'SEM' is the correct acronym of 'scanning electron microscopy.'

Q13 APT to AU: Please check that the edits to the preceding sentence are OK and that the meaning has not been changed.

Q14 APT to AU: Please define 'PS' at the first occurrence in the text.

Q15 APT to AU: Please provide volume and page numbers in ref. (11h) if now available.

Q16 APT to AU: Please provide the publisher name and its location in ref. (12).

Q17 APT to AU: Please provide the page number in ref. (20), if now available. 